\title{
Movimientos juveniles y políticas públicas de juventud en México: una aproximación conceptual
}

\section{Youth movements and public youth policies in Mexico: a conceptual approach}

\author{
Robert González García \\ Universidad Autónoma del Estado de Hidalgo, México \\ robert_gonzalez9475@uaeh.edu.mx \\ Código Orcid: https://orcid.org/0000-0002-6166-5562 \\ Juan A. Taguenca Belmonte \\ Universidad Autónoma del Estado de Hidalgo, México \\ juantaguenca@yahoo.com.mx \\ Código Orcid: https://orcid.org/0000-0002-9465-9951
}

\begin{abstract}
Resumen
En los últimos años emergen en México nuevas formas de participación política juvenil. Sin embargo, las políticas públicas de juventud siguen abordando la atención a las problemáticas juveniles sin su participación y de forma deficitaria. El objetivo del artículo es trazar un marco conceptual que permita pensar unas políticas públicas de juventud desde y para los mismos jóvenes mexicanos. La metodología es el análisis documental y la hermenéutica de los procesos sociales que protagonizan los jóvenes mexicanos en los ámbitos de la participación política en los últimos años, entrecruzándolo con el análisis de la respuesta de las administraciones en forma de políticas de juventud. Los resultados evidencian la emergencia de una juventud más participativa que, sin embargo, muestra más continuidades que cambios en términos de cultura política y que convive con la persistencia de políticas públicas juveniles propias de periodos anteriores. La conclusión es que caminar hacia unas políticas públicas juveniles participativas es un objetivo importante para la democratización y mayor eficiencia de la atención a los jóvenes.
\end{abstract}

\section{Palabras clave}

Cultura política, juventud, México, movimientos sociales, participación política, políticas públicas.

Forma sugerida de citar: González García, R., y Taguenca Belmonte, J. (2019). Movimientos juveniles y políticas públicas de juventud en México: una aproximación conceptual. Universitas, 31, pp. 37-57. 


\begin{abstract}
In recent years, new forms of youth political participation have emerged in Mexico. However, youth public policies continue to address the problems of the youth without their participation and in a deficient manner. The main objective of the article is to draw up a conceptual framework that allows us to think about public policies of youth from and for the young Mexicans themselves. The methodology is the documentary analysis and the hermeneutic of the social processes that star young Mexicans in the areas of political participation in recent years, intersecting it with the analysis of the response of administrations in the form of youth policies. The results show the emergence of a more participatory youth that, however, shows more continuities than changes in terms of political culture and that coexists with the persistence of youth public policies typical of previous periods. The conclusion is that moving towards participatory youth public policies is an important objective for the democratization and greater efficiency of the attention and service for young people.
\end{abstract}

Keywords

Political culture, youth, Mexico, social movements, political participation, public policies

\title{
Introducción
}

En el presente artículo se realiza un análisis de la participación ciudadana de la juventud mexicana y de las políticas públicas que atienden a sus necesidades, es decir, que los tienen como grupo social con características propias derivadas de su rango de edad, motivación biológica, que hace necesario su sujeción a acciones específicas de las instituciones gubernamentales. Esto se justifica mediante la división social por edades. Esta división particulariza determinadas problemáticas que afectan diferencialmente a jóvenes, adultos y adultos mayores. De ahí que se dé un tratamiento institucional específico para los jóvenes que se canaliza mediante subdivisiones sociales problematizadas: sexualidad, salud, trabajo, ocio, cultura y adiciones son algunos ejemplos de ello.

El artículo se centra en los jóvenes, la participación y la política pública de éstos. Estos tres elementos sirven de marco referencial para el artículo. Asimismo, se orienta hacia el análisis de las políticas públicas enfoca- 
das a la juventud. En este sentido, se adopta la perspectiva de la gobernanza o gobierno en red. Se parte del concepto de participación, entendiéndola como toda acción colectiva de individuos orientada a la satisfacción de determinados objetivos; y de política pública, definida como las acciones gubernamentales que son producto de una agenda pública que emana de las demandas ciudadanas. Es también importante señalar que se parte de la gobernanza participativa y de proximidad, en su visión normativa, como forma más democrática y eficiente de articular la participación ciudadana y las políticas públicas (Blanco \& Gomà, 2003).

En primer lugar, se revisará bibliografía relevante sobre la participación juvenil que ha tenido lugar en México en los últimos años, la cual refleja un resurgir de la implicación de los jóvenes mexicanos con la política. Se tomará en cuenta tanto la participación convencional (fundamentalmente electoral) como la no convencional (en especial la que se vehicula a través de los movimientos sociales), con el fin de detectar si existen cambios o continuidades en la cultura política juvenil mexicana y en qué sentido. Ello nos permitirá elaborar hipótesis que puedan ser contrastadas en futuras investigaciones. Se presenta la orientación conceptual elegida por los autores del artículo para abordar la conceptualización de la juventud.

En el segundo apartado, se presenta la emergencia de nuevos movimientos juveniles como el Yo soy 132 o el de solidaridad con la desaparición forzada de los 43 de Ayotzinapa, como contrapuntos a la desafección hacia los mecanismos de la democracia representativa tradicional.

El tercer apartado analiza si los cambios en la participación juvenil en México se han traducido o no en cambios sustantivos en términos de cultura política juvenil.

El cuarto apartado se dedica a abordar las políticas públicas mexicanas que tienen como objetivo a la juventud. Al respecto, se realiza una síntesis histórica partiendo de su surgimiento y analizando su desarrollo desde la teoría normativa de la política pública.

Los cuatro apartados permiten realizar un ejercicio comparativo entre la participación ciudadana juvenil y las políticas públicas que tienen como objetivo a la juventud, por un lado, con la gobernanza participativa y de proximidad, en su visión normativa, por el otro. Este ejercicio se retoma en las conclusiones.

En cuanto a la metodología utilizada, el análisis documental se define como: “..., un trabajo mediante el cual [...,] extraemos unas nociones del documento para representarlo [...] Analizar, por tanto, es derivar de un do- 
cumento el conjunto de palabras y símbolos que le sirvan de representación" (Rubio, s.f.). En nuestro artículo nos apoyamos de fuentes bibliográficas, principalmente teóricas, y en las categorías de análisis siguientes: Participación juvenil, desafección democrática, movimientos juveniles de protesta, YoSoy 132 y políticas públicas de juventud, todas ellas aplicadas a México.

Respecto a la hermenéutica de los procesos sociales, ésta la entendemos desde una reconstrucción permanente de los individuos de: "sus condiciones y sus mundos de la vida, [en la que aprenden] a reconocer y apropiarse de la infinidad de recursos construidos por la civilización en los diferentes dominios de la vida social. Recursos y dispositivos técnicos, y recursos y dispositivos simbólicos" (Vizer, 2007). Aquí se utilizaron fuentes bibliográficas teóricas y el análisis se implementó con base al procedimiento del socioanálisis, que "permite entender el territorio mediante un mapa de relaciones y estructuras de poder que determinan las interacciones entre los distintos agentes, instituciones y grupos sociales" (Villasante, 1999 en Pérez, 2011, p. 26). Al respecto, se realizó el esfuerzo de objetivar la posición de los jóvenes estudiados con respecto a la política.

\section{La participación juvenil en México}

Respecto al marco conceptual de la participación juvenil, la primera cuestión que se presenta es la de definir qué se entiende por juventud. Al respecto, existen estudios que consideran joven a aquella persona que se halla en una etapa posterior a la adolescencia, una etapa en la que el individuo ya puede iniciar su proceso de emancipación. En este sentido, la frontera con la niñez se establece en la edad legal para trabajar y manejar, que en México es a los 16 años.

Sin embargo, para analizar la participación electoral de los jóvenes es necesario considerar, por la parte baja de la horquilla, solo a los que tienen cumplidos los 18 años, puesto que antes de esa edad no se tiene el derecho político de votar en la mayoría de países, aunque se haya abierto un debate sobre la posibilidad de que el derecho a voto sea a partir de los 16 años (Ruiz de Azua, 2010). Finalmente, la Encuesta Nacional sobre Cultura Política 2012 (ENCUP) delimitó el rango de jóvenes a entre 18 y 29 años (Gómez, Tejera \& Aguilar, 2012). Teniendo en cuenta lo expuesto, utilizaremos este último rango para delimitar a los jóvenes mexicanos en su relación con 
la política. De esta forma, el criterio utilizado institucionalmente para la participación política es el de rango de edad, que contiene en sí el aspecto legal de inicio de un derecho político tan importante como es el ejercicio de elección de representantes en la esfera pública.

Lo anterior no es óbice para que en este artículo no nos posicionemos teóricamente. Existen en la actualidad varios enfoques teóricos sobre la juventud, de los cuales destacamos los siguientes, dada la importancia que han adquirido.

- Enfoque de la transición funcionalista. Se basa en los ciclos vitales, en la que la juventud es tratada "como una categoría social [delimitada por] criterios demográficos" (Brunet \& Pizzi, 2013, pp. 13-14). Es un enfoque que permite la administración de la juventud, al permitir su categorización social, haciendo posible "el ejercicio institucionalizado de políticas públicas singularizadas en rangos de edad concretos" (Taguenca, 2016, p. 186). Su variante es adulto-céntrica, ya que considera la juventud como una etapa previa a la edad adulta y en la que el joven se presenta como una especie de adulto incompleto, "que todavía tiene que madurar". Es una visión paternalista y que analiza a los jóvenes "en negativo", es decir, destacando todas aquellas características que les faltan para llegar a la etapa adulta.

- Enfoque de la transición biográfica. Se centra en la biografía de los individuos y tiene una posición culturalista muy influenciada por los estudios de identidad juvenil, y con poco desarrollo de las cuestiones materiales que afectan a los jóvenes. Este enfoque destaca la heterogeneidad de las trayectorias biográficas juveniles, las transiciones a las que éstos se enfrentan - por ejemplo: escuela-trabajo, hogar parental-hogar propio- y tres dimensiones: biográfica, institucional y socio-histórica, tal y como se muestra en los trabajos de Coleman y Husen (1985) y Furlong y Cartmel (1997).

- Enfoque nominalista. Recoge el trabajo previo de Mannheim (1993 [1928]), en el que la juventud solo constituye un grupo social cuando enfrenta idénticos acontecimientos en idénticos periodos de vida, y en el que el origen social adquiere especial relevancia, por ejemplo, desde la invención social (Galland, 2011) o como categoría socio-histórica (Allerbeck \& Rosenmayr, 1979 [1974]).

- Enfoque del conflicto generacional. Parte de las ideas de Bourdieu (2002 [1978]) sobre el conflicto que mantienen jóvenes y adultos en 
diferentes campos sociales, y que es consecuencia de diferentes estados sociales que conforman distintas producciones de sujetos diferenciadas por edad, con la consecuente división desigual del poder y demarcación sucesoria. Una variante de este enfoque es la del denominado radicalismo juvenil, que pone el énfasis en la ruptura generacional y en su potencial transformador.

De los enfoques vistos, nos decantamos por el de la transición biográfica, también conocida como la de los ciclos vitales. En esta se considera la infancia, la adolescencia y la juventud como tramos en el proceso biográfico de los sujetos hacia la toma de posición en los roles familiares y otros tramos vitales hasta la vejez y la posterior decrepitud (Casal, García, Merino \& Quesada, 2006). Su metodología específica es la de los itinerarios y las trayectorias sociales que se traduce a menudo en biografías. Se reconoce que el tramo de la juventud tiene unos determinantes estructurales e institucionales.

Señalado el enfoque de juventud que sirve de referente a este artículo, es hora de situar las perspectivas de abordaje de la cultura política y la participación política juvenil. Con relación a esto, hay que destacar, previamente, que en los últimos años el debate sobre el papel de los jóvenes en la política ha cobrado un inusitado interés debido a movilizaciones como: las del 15M, en España (Minguijón \& Pac Salas, 2013; Romero, 2015), Yo soy 132, en México (Brachet, 2014) y la Primavera Árabe (Blanco, 2011; Roque, 2015), que tuvieron lugar en 2011.

Estas movilizaciones han mostrado que los jóvenes han asumido, en diversos países y continentes, un papel protagónico en los procesos de cambio político (Gómez, Tejera \& Aguilar, 2012), desmintiendo el cliché de los jóvenes postmodernos como desafectos de la política, pasivos e interesados sólo en su hedonismo a través del consumo. Este cliché ha sido paulatinamente abandonado, y ha dado paso a múltiples estudios sobre la implicación juvenil en la política (Benedicto, 2008; Soler-i-Martí, 2014; Villa, 2015; Taguenca, González, et al., 2018).

Dado lo anterior, cabe preguntarse si realmente estamos ante un ciclo nuevo de participación política juvenil basado en un cambio de su cultura política, más cívica en términos de Almond y Verba (1963), y desde luego más reivindicativa en términos políticos.

No cabe duda que la realidad juvenil anterior a la caída del muro de Berlín (1989) era sustancialmente distinta a la actual. La consolidación del neo- 
liberalismo político y de la globalización económica, a las que las nuevas tecnologías de la información y la comunicación - internet, a través de las redes sociales, sobre todo- han favorecido en su difusión a escala planetaria, han cambiado radicalmente la esfera pública y privada, y la sicología de los individuos, transformando radicalmente, tanto material y simbólicamente como en sus principios y valores, a las sociedades y a los jóvenes que en ellas habitan. A esto hay que unir la pérdida de centralidad del mundo del trabajo, que va unida al consumo como nuevo centro de los individuos.

La adopción de un sistema de producción post-fordista se ha traducido en profundos cambios socioeconómicos y estructurales que afectan directamente a la juventud. Destacamos: el retraso en la emancipación juvenil, debido a la "universalización de la enseñanza superior"; el cambio en las relaciones afectivas, que han pasado de la estabilidad emocional a las relaciones "de entrada rápida y salida fácil"; el aumento de la flexibilidad laboral, convertida en precariedad laboral e informalidad; el incremento de la movilidad espacial, por motivos formativos y laborales, sobre todo; la proliferación de nuevas formas de participación juvenil basadas en la flexibilidad.

Con todo, el cambio más significativo se ha dado en el campo cultural, y tiene que ver con la sustitución paulatina de las tradicionales relaciones de producción, centradas en el mundo laboral, por otras relacionadas con consumo ejercido en el tiempo libre, que se ha convertido en el eje vertebrador de las identidades personales y colectivas. Es en este sentido en el que surge el concepto de "juventud ociosa" (González et al., 2004), el cual se ha utilizado como sinónimo de "juventud apática".

Todo este conjunto de transformaciones sociales ha comportado cambios importantes en la transición biográfica de los individuos, la cual pasó de ser lineal y casi determinista a ser abierta e incierta. Hablamos de biografía lineal para referirnos al modus vivendi predominante en la Edad Contemporánea, basado en la consecución de etapas vitales más o menos comunes para el conjunto de ciudadanos y ciudadanas que vendrían determinadas por la estabilidad (etapa formativa durante la adolescencia, incorporación al mercado laboral con un trabajo indefinido, apareamiento a través de un núcleo familiar tradicional). Por el contrario, el concepto de biografía abierta hace referencia a unas etapas vitales que ni son consecutivas ni son comunes a la mayoría (alargamiento de las trayectorias formativas hasta bien entrada la vida adulta, inestabilidad sentimental y formación de diferentes núcleos familiares a lo largo de la vida, cambio constante de trabajo debido a la fle- 
xibilidad laboral y cambios en la vivienda habitual). Estas biografías abiertas son más comunes en la Edad Postmoderna en la que estamos inmersos. A pesar del cambio sustancial enunciado, el de la transición biográfica de los individuos, que pasa de la linealidad cerrada a la apertura que produce incertidumbre, no debemos olvidar que las condiciones objetivas de salida siguen siendo determinantes y que, por tanto, el paso de las biografías lineales a las biografías abiertas no significa la posibilidad de un cambio de estatus o de un cambio social por sí mismo (Furlong, 2012).

La apertura de la transición biográfica puede conducir a un cambio de la cultura política de los jóvenes, haciendo de estos individuos más participativos y portadores de un cambio social en el que su participación en los asuntos políticos es más activa que la de anteriores generaciones.

La participación electoral de los jóvenes mexicanos, en los inicios de la segunda década del siglo XXI, nos sirve para corroborar la hipótesis anterior. Claro está que debemos tomar medidas metodológicas adicionales, puesto que los indicadores de los que disponemos no están exentos de algunos problemas. Por ejemplo, más del $70 \%$ de los jóvenes encuestados manifestaba que votaría por alguno de los candidatos en la elección presidencial de 2012, aunque el resultado de la misma (con una participación del 63\%) reveló que ese dato estaba algo sesgado (Gómez, Tejera \& Aguilar, 2012). No olvidemos que la participación en las elecciones mediante el voto es la forma más común de participación política de los jóvenes.

La participación electoral de los jóvenes es importante para lo que aquí estamos examinando, pues nos permite observar si es significativamente distinta a la de otras cohortes de edad y si se decanta por opciones políticas distintas, y también cuál es su tendencia.

Respecto a lo anterior, la literatura académica sobre comportamiento político señala que existen diferencias de voto por edad: los jóvenes votan más a partidos nuevos y radicales en sus planteamientos. También existe un efecto generacional en el voto por determinadas opciones, mismo que responde a la atmósfera del momento (Anduiza \& Bosch, 2004).

Para el caso de México, la mayoría de estudios no encontraban diferencias relevantes en la orientación del voto en función de la edad. Sin embargo, Moreno (2003) demostró que ligado al proceso de transición democrática se puede distinguir entre un votante de mayor edad, más rural y con menos formación - que sería la base electoral del Partido Revolucionario Institucional (PRI) - y otro votante más joven, urbano y con mayores nive- 
les de formación, que se decantaría hacia el Partido Acción Nacional (PAN) o el Partido de la Revolución Democrática (PRD). El nivel de ingresos seria variable diferenciadora entre estos dos últimos.

\section{Desafección democrática y nuevos movimientos juveniles de protesta en el México contemporáneo}

En la Encuesta Nacional de Cultura Política 2012, prácticamente el $90 \%$ de los jóvenes mexicanos manifestaban poco o nulo interés por la política. También el $90 \%$ afirmaban no haber participado nunca en ningún tipo de organización política ni movimiento social, ni siquiera en acción colectiva alguna. La encuesta no puede medir los efectos que pudiera haber tenido el movimiento YoSoy 132 en 2012 - o solo lo hace parcialmente - ya que fue levantada en diversas fases, aunque sólo algunas de ellas poco antes del mismo (González \& Hernández, 2017). Por otro lado, según la misma encuesta la familia es todavía el principal agente de socialización política de los jóvenes mexicanos.

Además, en términos de desafección y desconfianza hacia la clase política, el 90\% de los jóvenes encuestados consideran que existe corrupción, derroche económico en acciones no relevantes y coste excesivo de las mismas. El desinterés por la política institucional de los jóvenes mexicanos es evidente. Por ejemplo, en plena campaña de elecciones presidenciales de 2012 tan solo un $25 \%$ se habían informado sobre la misma. En cambio, los jóvenes entrevistados tuvieron información de los tópicos más importantes del movimiento YoSoy132 (Gómez, Tejera \& Aguilar, 2012).

En este punto es importante introducir qué significa la política para los jóvenes. Al respecto, en un estudio de 2006, Megías distingue entre diversas tipologías de joven en función de sus percepciones y orientación hacia la política. Cabría diferenciar dos grandes tipos de jóvenes, subdivididos a su vez en sub-grupos, lo que da como resultado cinco tipologías.

En primer lugar, se encuentran los jóvenes que muestran gran desinterés por la política, considerando que esta no es de su incumbencia o al menos no lo es todavía por su corta edad; un segundo grupo muestra rechazo hacia los mecanismos formales de la política y lo extiende hacia todo lo político; un tercero muestra desconfianza hacia la efectividad de la acción política y de los actores políticos; el cuarto y quinto grupo muestran interés hacia la 
política, pero unos con una clara adhesión al status quo (partidos, gobiernos e instituciones formales) y otros, al contrario, tienen un fuerte compromiso hacia la transformación social desde medios alternos a los partidos, priorizando los movimientos sociales y las estructuras no formales. Los jóvenes partidarios en México no son muchos. En 2016, de los 8.07 millones de afiliados a cinco partidos (PAN, PRI, PRD, Movimiento ciudadano y MORENA - Movimiento Regeneración Nacional-), sólo había un 15\% de jóvenes (Ollin, 2016). En cuanto a los activistas en movimientos sociales, si bien es difícil cuantificarlos, vamos a tomar el movimiento Yo soy 132 como ejemplo de este tipo de participación juvenil novedosa.

En el contexto de la contienda electoral del año 2012 — en el mes de mayo - irrumpió de manera inesperada y original un movimiento estudiantil en la Universidad Iberoamericana. El detonante de este estallido social tuvo lugar en el contexto de la asistencia del candidato a la presidencia del PRI, Enrique Peña Nieto a la Universidad Iberoamericana, cuando al concluir la sesión fue abordado por un grupo de estudiantes que le cuestionaron el apoyo desmedido que estaba recibiendo de los medios de comunicación (en particular de Televisa). Jóvenes estudiantes de escuelas privadas y públicas se unieron en la protesta y establecieron sus demandas, al tiempo que se manifestaron como un movimiento incluyente, que remite al principio zapatista de conformar un "Mundo en el que quepan todos los Mundos" (Díaz, 2013).

Se puede afirmar pues que en México se ha producido una eclosión particularmente significativa de nuevos movimientos sociales en los últimos años, entre los cuales destacaríamos: el Yo soy 132 (Rovira, 2014), las protestas por la desaparición de los 43 normalistas de Ayotzinapa (Hernández, 2015), la huelga del Instituto Politécnico Nacional y las movilizaciones feministas contra la violencia de género desde abril de 2016. El éxito de estos movimientos frente al comprobado fracaso de los mecanismos tradicionales de participación (como los partidos políticos) es especialmente relevante para analizar la participación juvenil.

Se trata de movimientos con una estructura muy flexible. La inexistencia de estructuras rígidas hace que cada joven encuentre su lugar en función de su voluntad de implicación. Lejos de la cultura del sacrificio, propia de los movimientos sociales clásicos e incluso de los nuevos movimientos sociales de los años 70. Estos movimientos, al igual que otros previos, como el zapatista, funcionan con un sistema de democracia directa: tienen una estructura asamblearia que permite que todo el que quiera participe en el proceso de toma de decisiones. Sin entrar en las contradicciones o problemas 
de este modelo de democracia directa a través de las asambleas; de entrada, la percepción que se llevan los que participan es que pueden tener un papel mucho más relevante y mayor capacidad de decisión que en las estructuras tradicionales de democracia representativa a través de los partidos políticos, que es menos flexible y con menor capacidad de participación en la toma de decisiones, sobre todo de los jóvenes.

Son, además, movimientos adaptados a los ritmos y formas de vida actuales: la convocatoria y proceso de información se hace vía electrónica, con lo que sólo hay presencia en los momentos clave de la toma de decisiones y de acción colectiva. Incluso el debate y la deliberación pueden ser también virtuales: a través de las redes sociales, los blogs y las plataformas virtuales (González \& Hernández, 2017).

Finalmente, los movimientos juveniles actuales funcionan con base en procesos identitarios parciales, sin exigir al joven que comparta con todos los demás miembros de su sociedad una visión global y coherente del mundo, con base en la cual actúe, lo cual favorece la participación. Además, el hecho de plantear la participación en torno a temas concretos que buscan soluciones inmediatas permite que personas con visiones del mundo completamente diferentes, pero que comparten la visión sobre este aspecto puntual, compartan espacios de participación. En síntesis, así como en los mecanismos tradicionales, el propio movimiento buscaba la coherencia de sus acciones y ofrecía a los individuos una cosmovisión, los nuevos mecanismos ofrecen visiones más parciales y diluyen las identidades colectivas tradicionales (clase, género, etnia, etc.). Es el propio individuo quien debe dotar a sus acciones de una coherencia subjetiva interna, y no la sociedad a través de sus instituciones la que las guiará mediante una globalidad holística conformada por dualidades confrontadas socio-históricamente. Lo importante aquí es destacar que el individuo queda agenciado en su participación política, al contrario que en el viejo modelo en el que la agencia pertenece a subconjuntos sociales muy amplios y en conflicto, que además están insertos en la lógica de la representación.

\section{Cambios y continuidades en la cultura política juvenil mexicana}

A pesar de la aparición de novedosos movimientos como el YoSoy132, en México hallamos todavía fuertes continuidades en lo que se refiere a la 
cultura política que se relaciona con el voto partidario. Aun así, todavía es pronto para saber si estos movimientos de crítica al sistema político formal pueden cristalizar en nuevas formas de hacer política desde la ciudadanía y al servicio de la misma. De hecho, la posibilidad de presentar candidaturas ciudadanas, que apenas ha iniciado en México, puede ser una ventana de oportunidad para estas experiencias. Por otro lado, los primeros análisis sobre el espectacular cambio electoral que ha supuesto la victoria de Andrés Manuel López Obrador y de MORENA en las elecciones 2018, apuntan hacia una participación importante de los jóvenes en este vuelco político (Consulta Mitofsky, 2018).

En todo caso, por lo que se refiere a los partidos y al voto en elecciones presidenciales o legislativas, en México los partidos se han adaptado a la cultura política del PRI después de los cambios que permitieron la competencia y la alternancia electoral. Se podría decir que todos aprendieron a jugar con las mismas reglas de la cultura de los 70 años de partido hegemónico (Gómez, Tejera \& Aguilar, 2012). Ni siquiera las nuevas formaciones políticas como Movimiento Ciudadano, MORENA o el Partido Humanista, han aportado novedad alguna a las prácticas clientelares y corporativas de PRI, a las que también son afectos el PAN y el PRD.

En la investigación realizada encontramos las siguientes paradojas: si bien los jóvenes desconfían mayoritariamente de los políticos y rechazan la intermediación como forma de solucionar los problemas, al mismo tiempo no dudan en mostrar predilección por alguno de los candidatos en unas elecciones presidenciales. Por otro lado, según datos de la ENCUP 2012, los jóvenes mexicanos se manifiestan mayoritariamente indefinidos $(32 \%)$ y de derecha (32\%), pero en cambio sus posicionamientos político-ideológicos sobre determinados temas (privatización de PEMEX, estado del bienestar, etc.) los sitúan mayoritariamente en el campo de la izquierda (González \& Hernández, 2017).

La cultura política juvenil mexicana presenta desfases importantes y no pocas contradicciones. Los jóvenes dicen que son de derecha, pero manifiestan opiniones de izquierda; desconfían de los políticos, pero muestran preferencias claras por los distintos candidatos en unas presidenciales. Según Gómez, Tejera y Aguilar (2012) una posible explicación de estas contradicciones puede ser la incidencia de redes familiares y sociales en los posicionamientos sobre los partidos políticos, las instituciones electorales y el gobierno. 
En definitiva, los jóvenes mexicanos no están emancipados en términos de cultura política y están fuertemente influenciados por las generaciones precedentes, lo que nos remite a la hipótesis de que la socialización es una variable explicativa de la cultura política. Respecto a la socialización, Cot y Mounier (1978, p. 280 citado por Smith-Martins, 2000, p. 94) refieren que:

En la perspectiva de un sistema político establecido, la función de la socialización política consiste en garantizar la continuidad del sistema político a través de las generaciones. Por intermedio de la socialización, el sistema inculca a las nuevas generaciones los valores y el comportamiento político de las generaciones precedentes, a fin de garantizar la reproducción permanente.

La sociedad mexicana experimenta más continuidades que cambios en términos de cultura política y participación juvenil, quizá porqué el cambio institucional democrático no ha dado lugar al cambio de cultura política correspondiente. Está por ver si de los movimientos estudiantiles mexicanos, que históricamente han aportado dinamismo y cambio a la esfera política, pueden surgir iniciativas que desborden la cultura política dominante, caracterizada por sus rasgos autoritarios (Hernández, 2008).

\section{La emergencia de las políticas públicas de juventud en México}

La emergencia de las políticas de juventud se produce en paralelo a la imposición del modelo de globalización neoliberal y de gobernanza en todo el mundo. Pero la globalización no es un fenómeno que se imponga de manera absoluta, no representa un movimiento en un solo sentido, sino que suscita movimientos contrarios, que compensan, de algún modo, sus efectos perversos. El primero de los movimientos identificados es el de la tendencia al fortalecimiento de los poderes locales, como espacio de construcción de proyectos colectivos que profundizan la ciudadanía. Algunos politólogos se han referido a este movimiento como un proceso de politización de la esfera local (Brugué \& Gomà, 1998).

Los gobiernos locales parecen abandonar sus roles operativos tradicionales y su posición residual en los procesos de gobierno. Por otro lado, las agendas locales se amplían hacia campos emergentes de política pública, como en el caso de la juventud, y hacia ámbitos más tradicionales, como ocurre con la educación y el empleo. 
El concepto de gobernanza expresa una transformación general, la de los modos de articular los procesos de gobierno en la sociedad postindustrial, y la emergencia de nuevas formas de articular el conflicto social en el espacio local. Este apartado hace un recuento sintético de las etapas de las políticas de juventud que surgen y se desarrollan en México de forma paralela a estos procesos.

En México, los programas oficiales que tienen como objetivo a la juventud inician en la primera mitad del siglo XX, y se deben a las condiciones internacionales referidas a: la expansión del sistema capitalista, el nuevo ordenamiento geopolítico mundial, definido en las dos guerras mundiales, la explosión demográfica y los complejos procesos de urbanización e industrialización, así como por las consecuencias sociales y culturales que de todo ello derivan, y que se encuentran en estrecha relación con la diversificación económica y cultural de las sociedades occidentales.

Los años comprendidos entre las décadas de 1930 y 1950 representan el reconocimiento explícito, por parte de los gobiernos occidentales, de la existencia de la juventud como categoría separada, con problemáticas propias, de los niños y de los adultos. Esto hace que se realicen esfuerzos para definirla de forma objetiva y operativa, a fin de establecer políticas institucionales específicas (Marcial, 2012).

Se pueden establecer, con fines analíticos, diversas etapas de las políticas de juventud en México, teniendo en cuenta para ellos los cambios en las relaciones entre gobierno y juventud. Pasamos a continuación a detallarlas sintéticamente.

lera etapa. 1938-1949. "Surgimiento de los jóvenes". Esta primera década se caracterizó por la reducción de las políticas de juventud a las políticas educativas diseñadas a atender las problemáticas de los estudiantes universitarios. En este periodo, destaca un agente no gubernamental, la Iglesia Católica, quien desde su posición institucional privilegiada con la educación y el ocio de los jóvenes propicia una socialización de la juventud mexicana centrada en la obediencia y subordinación a los valores adulto-céntricos.

2 da etapa. 1950-1988. Atención integral y cooptación. En 1950, el presidente Miguel Alemán promulgó el decreto de fundación del Instituto Nacional de la Juventud Mexicana (INJM), a través del cual se pretendía dar capacitación laboral, cultural, ciudadana y física a los jóvenes mexicanos (Marcial, 2012). En este periodo, las políticas del INJM se centraron mayoritariamente en el Distrito Federal (D.F.) y a algunas pocas zonas rurales. Los programas del INMJ se utilizaron como mecanismo de cooptación de 
jóvenes, persiguiendo como objetivo específico no declarado la supresión del movimiento estudiantil, en especial en los años 60 y 70. En el sexenio de Luis Echeverría (1970-1976), el INJM cambió de nombre y pasó a denominarse Instituto Nacional de la Juventud (INJUVE). Con el cambio de nombre se puso de manifiesto la voluntad gubernamental de abrirse a las diversas expresiones juveniles. A pesar de esas intenciones, la realidad de las políticas gubernamentales siguió siendo represiva respecto al movimiento estudiantil y a los contenidos culturales propiamente juveniles, como es el caso de los conciertos de rock. Por otro lado, en este periodo la ciudad de Guadalajara fue el escenario de los principales enfrentamientos entre guerrilla urbana y grupos porriles - agrupaciones juveniles de choque patrocinadas por los gobiernos o, incluso, las rectorías universitarias, cordón de aquellos en el caso de las universidades estatales mexicanas- y paramilitares (Aguayo, 2001, p. 170).

En el sexenio de López Portillo (1976-1982), con la intención de organizar a los jóvenes "desde arriba", se conforma el Consejo Nacional de Recursos para la Atención de la Juventud (CREA). A pesar de su diseño jerárquico, por primera vez se piensa a los jóvenes más allá de las aulas y se establece una política social juvenil. Con el CREA se empieza a adoptar una perspectiva de gobernanza, al implicar a la sociedad civil y a los jóvenes (Pérez-Islas, 2000). La 2da etapa finaliza en 1987-1988 con la descentralización estatal, municipal y regional del CREA. Destacar que en todo este periodo, el deporte y el turismo actúan como límites de la política de juventud; y que, a pesar de la agenda más social y la descentralización, permanece la estrategia de cooptación de los jóvenes por parte del sistema político hegemónico priista, como demuestra el caso del Consejo Popular Juvenil del DF (Marcial, 2012).

3era. Etapa. 1988-1999. La década perdida. Después del fraude electoral de 1988, el Gobierno de Salinas de Gortari impone con mano de hierro la doctrina neoliberal. Para las políticas de juventud esto significa reduccionismo deportivo y recortes presupuestarios. El neoliberalismo que se instala en la política gubernamental hasta nuestros días supone, en la práctica, el abandono y desinterés gubernamental por la juventud. A pesar de esta tónica general, en 1996, ya en el sexenio de Zedillo, el programa Causa Joven implica el reconocimiento de la diversidad juvenil. Siempre desde una perspectiva adultocrática, se proponen medidas de control y corrección, que corresponden a la visión adulta predominante que considera a los jóvenes como un 
problema. Finalmente, en 1999 con el nacimiento del Instituto Mexicano de la Juventud (IMJUVE), y no por casualidad en coincidencia con la Huelga de la UNAM, se retoman las políticas de juventud.

4ta Etapa.2000-2006. La atención selectiva. En el año 2000 se produce por primera vez en 70 años la alternancia en la presidencia de la República, con la victoria electoral de Vicente Fox, candidato apoyado por el PAN. En este sexenio se pretendió dar continuidad a las políticas de juventud iniciadas por IMJUVE. Sin embargo, se observa como en el transcurso del mismo se produce un deslizamiento hacia la "derecha" neoliberal y globalizadora en las políticas de juventud (Marcial, 2012). En este sexenio se inicia el Programa Nacional de Juventud, un mecanismo de participación centralizado y controlado "desde arriba". También se responde al cambio demográfico y social con la ampliación - por arriba y por abajo - de las edades comprendidas en el concepto de juventud, que queda comprendida entre los 12 y los 29 años. Finalmente, también inicia en este periodo un excelente, aunque a menudo infrautilizado instrumento, la Encuesta Nacional de Juventud.

5ta Etapa. 2006-2018. Jóvenes a la deriva: desatención y represión. Tanto el sexenio de Calderón (PAN), 2006-2012, como el de Peña Nieto (PRI), 2012-2018, suponen un regreso del partidismo y la captura de la red de políticas de juventud por parte del Gobierno. Las viejas prácticas autoritarias de uso de los organismos juveniles, como los mecanismos de control y represión de los movimientos de jóvenes, utilizadas ya en los años setenta, regresaron; y lo hicieron en medio de la acentuación del programa neoliberal y su consecuente abandono de las políticas estructurales dirigidas a los jóvenes (Macías, 2012).

El profundo cambio político producido en las elecciones de 2018 con la victoria por primera vez en México de la oposición de izquierda en todos casi los ámbitos de gobierno (Presidencia, Congreso, Senado y la mayoría de congresos locales) abren la posibilidad de una sexta etapa, donde a priori la participación de los jóvenes en el diseño, implementación y evaluación de políticas de juventud, debiera ser más activa.

\section{Conclusiones y propuestas. Hacia unas políticas públicas de juventud participativas}

La mayor parte de la historia de las políticas públicas juveniles en México se ha caracterizado por unas políticas de juventud sin una extensa y cua- 
litativa participación de los jóvenes. Los cambios sociales y económicos de las últimas décadas, así como la emergencia de una juventud más activa con expresiones como el movimiento YoSoy132, abren la posibilidad de unas políticas de juventud participativas que se inserten en procesos de gobierno multinivel, es decir, de todas las escalas de gobierno - desde la federal hasta la municipal, pasando por la estatal - con mecanismos de confluencia en buena parte de las actuaciones públicas de juventud. Por otra parte, la implicación de los jóvenes mexicanos en política en los últimos años muestra que para que haya política de juventud debe haber acción desde y con los jóvenes, ya no valen criterios predominantemente tecnocráticos y acciones para los jóvenes.

La idea y el discurso de la participación están presentes en los últimos tiempos en México, que no en vano presidió la Alianza para el Gobierno Abierto en 2015. Ahora bien, la instrumentación de la participación se ha hecho de forma poco satisfactoria, apoyada en una marginación de los jóvenes del ámbito de la participación política. El modelo de los Institutos de Juventud, como esquema de participación estrictamente asociativa o partidista, con poca influencia en la definición de problemas y la toma de decisiones, y con un funcionamiento intermitente y bastante centralizado, es un modelo que hay que dar claramente por superado. En el contexto de los procesos de replanteo participativo que están haciendo hoy algunos municipios y en el contexto de los nuevos instrumentos de innovación democrática local, es posible plantear algunas pistas de por dónde deben ir los nuevos esquemas de participación juvenil.

En este artículo se han planteado elementos conceptuales novedosos para pensar unas políticas de juventud centradas en el propio sujeto juvenil y su capacidad de agencia. En primer lugar, las características de los movimientos juveniles que han emergido en México en la última década, tales como la estructura flexible y predominantemente horizontal, el uso de las nuevas tecnologías o las identidades fragmentadas y parciales, sirven de base conceptual para unas nuevas políticas públicas basadas en la participación juvenil. Asimismo, los cambios socioculturales en el marco del capitalismo posfordista que inciden en las trayectorias biográficas de los jóvenes - convirtiéndolas en flexibles, no lineales y continuamente adaptables al cambio - afectan también a los sentidos y orientaciones de las políticas públicas de juventud en México. 
En consecuencia, las políticas públicas de juventud necesitan desbordar la participación asociativa y partidista formal para implicar a jóvenes no organizados y a los activos en movimientos sociales y comunitarios. En segundo lugar, deben garantizar su relevancia y eficacia, es decir, convertirse en los espacios centrales de definición de políticas de juventud, introduciendo la participación y el uso de las nuevas tecnologías en todas las etapas de la política pública, desde la definición del problema, al diseño, la implementación y la evaluación de las mismas. En tercer lugar, requieren arraigarse en las dinámicas de las comunidades juveniles, mucho más flexibles y adaptables, lejos de la rigidez y aversión al cambio del centralismo institucional. Estos criterios normativos serán elementos para próximos análisis empíricos que no caben en este artículo, centrado en exponer el tema y aportar un marco conceptual y analítico. De momento, con lo descrito, queda mucho margen de mejora para las políticas juveniles en México.

\section{Bibliografía}

Aguayo, S. (2001). La Charola. Una historia de los servicios de la inteligencia en México. México DF: Grijalbo.

Almond, G., \& Verba, S. (1963). The Civic Culture: Political Attitudes and democracy in Five Nations. Newbury Park: Sage Publications.

Allerbeck, K., \& Rosenmayr, 1. (1979 [1974]) Introducción a la sociología de la juventud. Buenos Aires: Kapelusz.

Anduiza, E., \& Bosch,A. (2004). Comportamiento político y electoral. Barcelona: Ariel.

Benedicto, J. (junio de 2008). La juventud frente a la política: ¿desenganchada, escéptica, alternativa o las tres cosas a la vez? Revista de estudios de juventud, 81, 13-28. Recuperado de: https://bit.ly/2NGSreD (2019-07-25).

Blanco, J. M. (2011). Primavera árabe. Protestas, y revueltas. Análisis de factores. Instituto Español de Estudios Estratégicos. Recuperado de: https://bit.ly/2nUD5dq (2019-07-20).

Blanco, I., \& Gomà, R. (2003). La crisis del modelo de gobierno relacional: Reflexiones en torno a la governance participativa y de proximidad, Gestión y Política Pública, 12(1), 5-42.

Bourdieu, P. (2002 [1978]) La juventud no es más que una palabra. En: P. Bourdieu, Sociología y cultura. México D.F.: Grijalbo y CONACULTA. 
Brachet, V. (2014). Sistema de protesta: política, medios y el \#YoSoy 132. Sociológica, 29(82), 83-123. Recuperado de: https://bit.ly/32YmF5B (201907-23).

Brugué, Q. \& Gomà, R. (1998). Gobiernos locales y políticas públicas. Barcelona: Ariel.

Brunet, I. \& Pizzi, A. (2013). La delimitación sociológica de la juventud. Última Década, (38), 11-36.

Casal, J., García, M., Merino, R. \& Quesada, M. (2006). Aportaciones teóricas y metodológicas a la sociología de la juventud desde la perspectiva de la transición, Papers, 79, 21-48. Recuperado de: https://bit.ly/2SNJqUX (2018-09-23).

Consulta Mitofsky (2018). México: encuestas de salida 4 elecciones presidenciales 2000-2018. Recuperado de: https://bit.ly/2YcRBAe (2018-11-15).

Coleman, J. \& Husen, T. (1985). Becoming Adult in a Changing Society. París: OCDE.

Díaz, A. S. (2013). Quisieron arrebatarnos todo y lo único que perdimos fue el miedo: el espacio zapatista y sus principios en el movimiento YoSoy132 de México. Revista interdisciplinaria de ciencias sociales, 7, 101-127.

Furlong, A., \& Cartmel, F. (1997). Young people and Social Change: Individualisation and Risk in the Age of High Modernitity. Buckingham: Open University Press.

Furlong, A. (2012). Youth Studies: an introduction. London: Routledge.

Galland, O. (2011) Sociologie de la jeunesse. Paris: Armand Colin.

Gómez, S. Tejera, H., \& Aguilar, J. (2012). Informe de la Encuesta "La cultura política de los jóvenes en México” para el Instituto Federal Electoral. México: Colegio de México.

González, R., Gomà, R., et al. (2004). Joventut, okupació i polítiques públiques a Catalunya. Barcelona: Secretaria General de la Joventut, Generalitat de Catalunya.

González, R., \& Hernández, J. E. (2017). La participación política juvenil en México y España. Una perspectiva comparada. En B. Lugo y J.A. Taguenca, Democracias. Una panorámica (pp. 173-200). Pachuca de Soto: UAEH.

Hernández, L. (2015). Ayotzinapa: el dolor y la esperanza. El Cotidiano, 7-17. Recuperado de: https://bit.ly/2GCRYJx (2019-07-26).

Hernández, M. A. (2008). La democracia mexicana, presa de una cultura política con rasgos autoritarios. Revista Mexicana de Sociología, 70(2), 261-303. Recuperado de: https://bit.ly/2Yodlo4 (2018-09-25). 
Mannheim, K. (1993 [1928]) El problema de las generaciones. Revista Española de Investigaciones Sociológicas, 62, 193-242. Recuperado de: https://bit. ly/2ynXiw2 (2018-10-18).

Megías, E. (2006). Jóvenes y política. El compromiso con lo colectivo. Madrid: FAD. Minguijón, P., \& Pac Salas, D. (2013). La primavera española del 15 M. Política y gobierno, 20(2), 359-389. Recuperado de: https://bit.ly/2EyxBgc (201907-24).

Moreno, A. (2003). El votante mexicano. Democracia, actitudes políticas y conducta electoral. México: Fondo de Cultura Económica.

Ollin, Jóvenes en Movimiento, A.C. (2016). Índice de Participación Juvenil 2015 2016. México D.F: Ollin, Jóvenes en Movimiento, A.C.

Pérez, D. A. (2011). La hermenéutica y los métodos de investigación en ciencias sociales. Estudios de filosofía, 44, 9-37. Recuperado de: https://bit. ly/2MnXFik (2019-07-27).

Pérez-Islas, J. A. (Coord.) (2000). Jóvenes e Instituciones en México, 1994-2000. Actores, políticas y programas. México DF: Instituto Mexicano de la Juventud.

Roque, M. Á. (2015). Los jóvenes en la Primavera Árabe. Ankulegi, 19, 11-24. Recuperado de: https://bit.ly/2KaNjj9 (2019-07-26)

Romero, A. (2015). Historia de un movimiento. El 15-M como expresión del malestar social. Nómadas. Revista Crítica de Ciencias Sociales y Jurídicas, 46(2), 61-84. Recuperado de: https://bit.ly/2LMAKO8 (2019-07-26).

Rovira, G. (2014). El \#Yo Soy 132 mexicano: la aparición (inesperada) de una red activista. Revista CIDOB d'Afers Internacionals, 105, 47-66. Recuperado de: https://bit.ly/2yq8qbq (2019-07-27).

Rubio, M. C. (s.f.). El análisis documental: Indización y resumen en bases de datos especializadas. Recuperado de: https://bit.ly/2hyvL64 (2019-07-26).

Ruiz de Azua, M. A. (2010). La larga marcha hacia la ampliación del derecho de sufragio y el tema de la edad. En INJUVE, documentos. Recuperado de: https://bit.ly/1EZscs4 (2018-09-25).

Smith-Martins, M. (2000). Educación, socialización política y cultura política. Algunas aproximaciones teóricas, Perfiles educativos, 22(87), 76-97.

Soler-i-Matí, R. (2014). Youth political involvement update: measuring the role of cause-oriented political interest in young people's activism. Journal of Youth Studies, 18(3), 396-416. https://doi.org/10.1080/13676261.2014.963538.

Taguenca, J.A. (2016). Sociología de la juventud. Una revisión, Espacio Abierto Cuaderno Venezolano de Sociología, 25(3), 183-195. Recuperado de: https://bit.ly/2LNEHlz (2018-09-25). 
Taguenca, J. A., González, R., Rodríguez, M., \& Segura, T. (2018). Bosquejo de las identidades juveniles en México: Fragmentación y multiplicidad a la luz de sus contextos glocales. En C. Váquez, M. C. Ospina-Alvarado, y M. I. Domínguez, Juventudes e infancias en el escenario latinoamericano y caribeño actual (pp. 135-154). Buenos Aires: CLACSO.

Villa, P. C. (2015). Los jóvenes y la participación política en los contextos actuales: Las disputas en el espacio público mediatizado de hoy. Recuperado de: https://bit.ly/2ynoyuu (2019-07-25).

Vizer, E. A. (2007). Modelización del conocimiento social: la comunicación como estrategia de aproximación expresiva de los mundos sociales. Proposiciones para un programa de investigación sociocomunicacional. Razón y palabra, 59. Recuperado de: https://bit.ly/2Kf5F2g (2019-07-26).

Fecha de envío: 2018/12/04; Fecha de aceptación: 2018/07/29;

Fecha de publicación: 2019/09/01 